\title{
Outcomes of vitrectomy in severe complications of proliferative diabetic retinopathy
}

\author{
Dorota Borowicz, Dominika Nowakowska, Edyta Koman, Katarzyna Nowomiejska, Robert Rejdak
}

Department of General Ophthalmology, Medical University in Lublin, Lublin, Poland

\begin{abstract}
INTRODUCTION. The aim of our study is to describe the anatomical and functional results of pars plana vitrectomy (PPV) among diabetic patients treated due to tractional retinal detachment (TRD) or non-clearing vitreous haemorrhage (VH).

MATERIALS AND METHODS. A retrospective analysis was made of clinical data of 45 eyes treated with 23G PPV for TRD (17 eyes) or VH (28 eyes). Preoperative and final visual outcomes and postoperative complications were recorded. The mean follow-up period was 12 months.

RESULTS. The postoperative visual acuity (VA) improved significantly in $60 \%$ of eyes, but remained unchanged or was worse in 40\%. Improvement of VA was more pronounced in the VH group. PPV was performed with silicone oil tamponade in $66.5 \%$, air tamponade in $29 \%$, and gas tamponade in $4.5 \%$. Postoperative complications included: retinal re-detachment (seven eyes), secondary glaucoma (nine eyes), and recurrent VH (two eyes).

CONCLUSIONS. PPV is an effective treatment method of complications of severe proliferative diabetic retinopathy inter alia the TRD and VH. Because surgical management of TRD and VH is demanding, anatomical and functional results are limited by complications.
\end{abstract}

KEY WORDS: proliferative diabetic retinopathy, vitreous haemorrhage, tractional retinal detachment, vitrectomy

Ophthalmol J 2017; Vol. 2, No. 1, 17-21

\section{INTRODUCTION}

Diabetes mellitus is a common chronic metabolic disease wherein the frequency of morbidity increases. The common complications of severe proliferative diabetic retinopathy (PDR) include vitreous haemorrhage $(\mathrm{VH})$, as well as tractional retinal detachment (TRD). Both are indications for vitreoretinal surgery [1].

The concept of pars plana vitrectomy (PPV) began in 1971 by Machemer [2] and was first introduced with $20 \mathrm{G}$ instrumentation in 1972 by O'Malley and Heintz [3]. Since then, the surgical instrumentation has been permanently improved. In 2005, Eckardt developed the $23 \mathrm{G}$ instrumentation [4]. Vitrectomy instruments are now available also in 25 and 27 gauge, which are better for navigation. The cutting speeds are increasing (up to 7500-8000 cuts per minute), which allows extremely precise cutting. The combination of high cutting speeds and small gauge instruments allows successful surgery in severe complications of PDR. PPV gives the possibility of removal of the fibrovascular tissue and blood from the vitreous cavity [5, 6]. However, there is a risk of surgically induced complications, such as bleeding and $\mathrm{VH}$, iatrogenic retinal breaks, recurrent $\mathrm{RD}$, and glaucoma.

This article describes the evaluation of the results and complication rates among diabetic patients treated with PPV due to TRD or VH.

\section{MATERIALS AND METHODS}

A retrospective analysis was performed on 45 eyes of 41 patients with PDR, who underwent surgery for the complications of PDR, TRD, or VH, in the Department of General Ophthalmology of Medical University of Lublin in Poland. The surgeries were conducted between January 2012 and December 2015. 

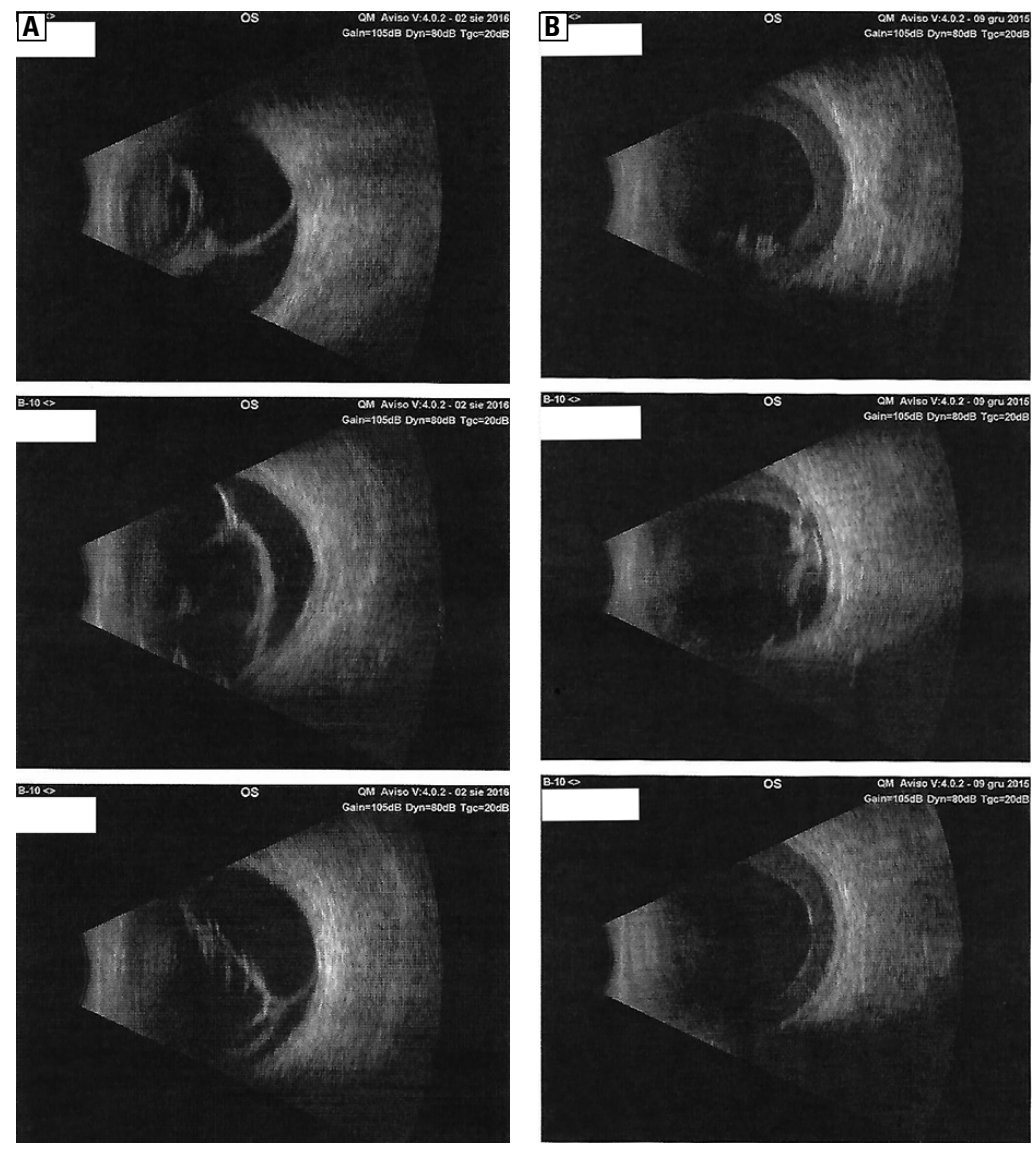

FIGURE 1. Tractional retinal detachment (A); vitreous haemorrhage in ultrasonography $(\mathbf{B})-B$ scan

All the patients were informed about the possible complications and everyone signed the agreement. The inclusion criteria were as follows:

- presence of TRD or

- non-clearing VH for more than three months.

Collected medical data were as follows: the type of diabetes, the duration of diabetes, the rubeosis iridis or the cataract presence, type of the tamponade used during PPV, preoperative and final best corrected visual acuity (BCVA), and postoperative complications, e.g.: secondary glaucoma, recurrent $\mathrm{TRD}$, or recurrent $\mathrm{VH}$. The main result measures were final visual outcomes and surgical complications. Each patient underwent a full ophthalmic examination, including BCVA testing with the Snellen method, slit lamp examination, measurement of intraocular pressure, and ultrasonography type B (Fig. 1). Snellen visual acuity records were converted to the logarithm of the minimum angle of resolution (LogMAR) value for statistical analysis.

The schedule of postoperative examinations was as follows: first day, 2 weeks, 6 weeks, and 4, 6, 9, 12,15 , and 18 months after surgery.
PPV is gaining popularity in the treatment of surgical vitreoretinal conditions because it may give faster recovery, but it requires a conjunctival peritomy.

Statistical analyses were performed using GraphPad Prism 5 software.

\section{SURGICAL TECHNIQUE}

Standard 23 G PPV was performed in local or general anaesthesia using the Constellation system (Alcon, Fort Worth, Texas, US). Complete PPV with assistance of triamcinolone and scleral indenting was performed. In order to avoid retinal damage, posterior vitreous detachment was not performed on the posterior pole. ILM peeling after indocyanine green (ICG) was performed if tractions were present in the posterior pole. The tractional membranes were removed bimanually using delineation and segmentation techniques. Panretinal laser coagulation was performed intraoperatively in each case. At the end of surgery, air, gas, or silicone oil was used as a tamponade. Sclerotomies were checked for leakage, and if needed, a Vicryl suture was applied (Fig. 2). 

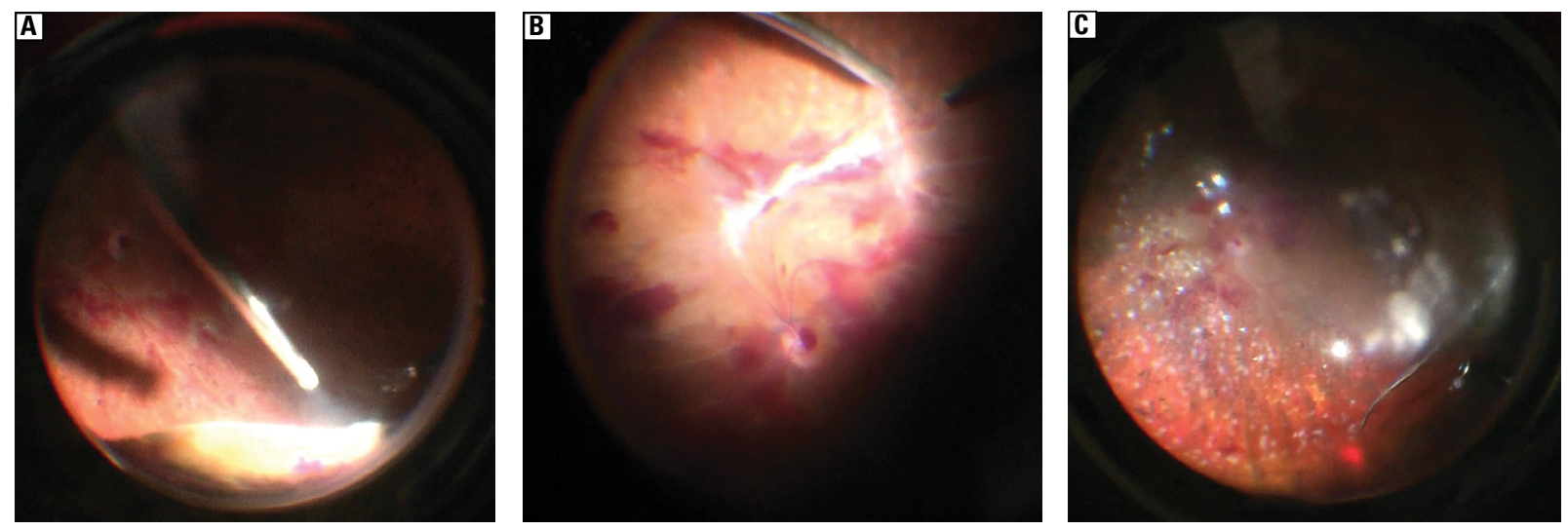

FIGURE 2. Intraoperative photographs: A. Shaving the vitreous base during the vitrectomy; B. Removing fibrovascular tissue in the posterior pole; C. Photocoagulation of the peripheral retina

\section{RESULTS}

The study included 45 eyes of 41 patients with complications for PDR: TRD (17 eyes, i.e. 38\%) or $\mathrm{VH}$ (i.e. 28 eyes, i.e. $62 \%$ ). The cohort included 23 men (57\%) and 18 women (43\%) aged from 25 to 84 years. Eight $(20 \%)$ patients were diagnosed with type I diabetes and $33(80 \%)$ patients with type 2 diabetes. The mean duration of diabetes was 14.5 years (range 1-34 years). Neovascularisation of the iris was recognised in eight eyes (18\%). Laser endophotocoagulation was performed in all eyes before PPV. Preoperative BCVA was between $20 / 40$ and 20/400 in 13 eyes (29\%), counting fingers in 10 eyes (22\%), and hand movements or light perception in 22 eyes (49\%). The mean follow-up period was 12 months. In 11 patients (i.e. 26\%) an intraocular lens was implanted during PPV (one patient had aphakia), and 30 patients (73\%) had pseudophakia before PPV. In 12 cases (27\%) ILM peeling was performed after dying with ICG during PPV. The primary PPV was performed with gas tamponade in two eyes (i.e. $4.5 \%$ ), silicone oil tamponade in 30 eyes (i.e. 66.5\%), and air tamponade in 13 eyes (i.e. 29\%). The postoperative BCVA improved significantly in 27 eyes $(60 \%)$ and remained unchanged or was worse in 18 eyes (40\%); however, visual acuity was hand movements or light perception in these eyes. The preoperative $\log$ MAR BCVA changed from $1.64 \pm 0.07$ to $1.41 \pm 0.08$ postoperatively (Fig. 3). The preoperative logMAR BCVA changed from $1.71 \pm 0.12$ to $1.69 \pm 0.14$ postoperatively among patients with I type diabetes. The preoperative logMAR BCVA changed from $1.80 \pm 0.09$ to $1.40 \pm 0.10$ postoperatively among patients with II type diabetes (Fig. 4). The preoperative logMAR BCVA changed

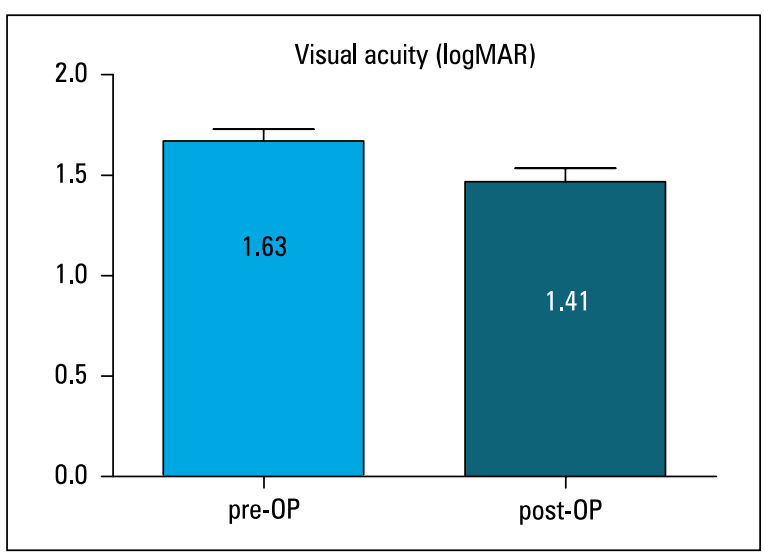

FIGURE 3. Postoperative (post-OP) versus preoperative (pre-OP) visual acuity (LogMAR)

from $1.39 \pm 0.66$ to $1.26 \pm 0.56$ postoperatively among patients with TRD. The preoperative $\log$ MAR BCVA changed from $1.90 \pm 0.07$ to $1.65 \pm 0.12$ postoperatively among patients with $\mathrm{VH}$ (Fig. 5).

IOP was elevated after PPV in nine eyes (eight eyes with silicone oil tamponade and one eye with gas tamponade) and was managed with topical antiglaucomatous drugs. Secondary TRD was observed in seven eyes after PPV with silicone oil tamponade, and in two cases re-VH appeared in patients with silicone oil tamponade.

\section{DISCUSSION}

Diabetes is a systemic disease with many systemic vascular complications. Glycaemic, blood pressure and cholesterol control should be optimised before surgery with the internist. Prolonged hyperglycaemia in diabetes leads to an increased permeability 


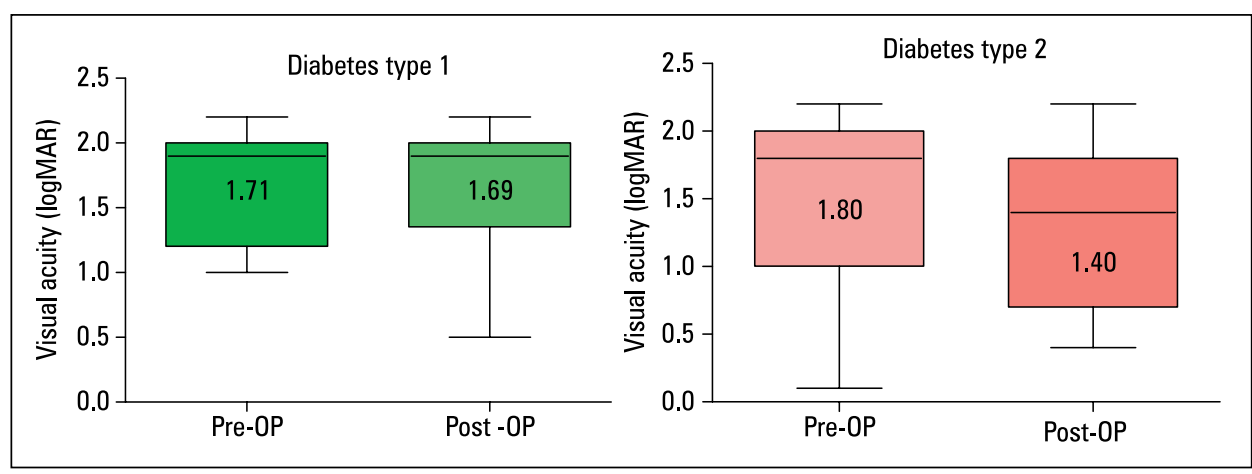

FIGURE 4. Comparison of preoperative (pre-OP) and postoperative (post-OP) visual acuity depending on the type of diabetes (type 1 and 2)

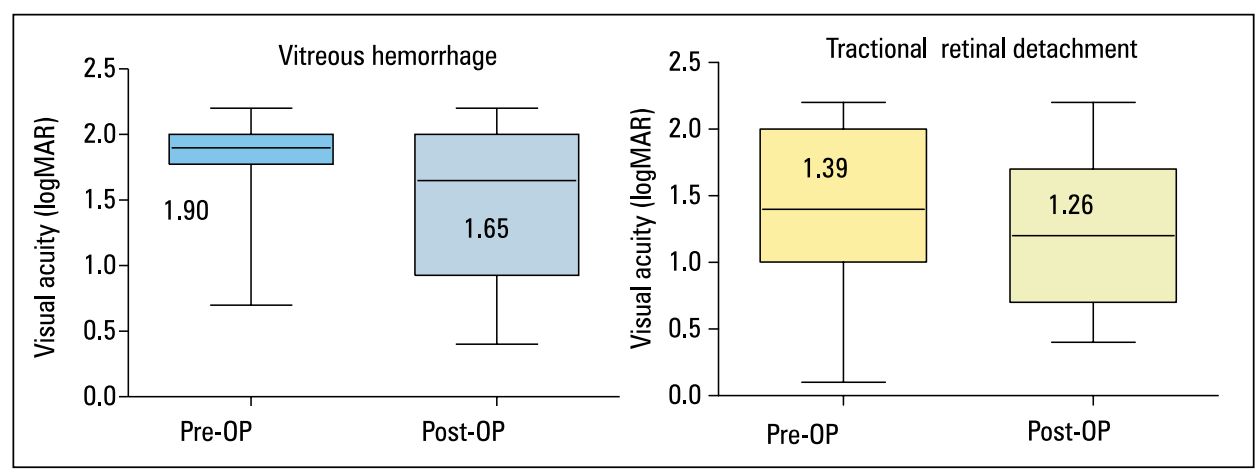

FIGURE 5. Comparison of preoperative (pre-OP) and postoperative (post-OP) visual acuity in two groups: with vitreous haemorrhage and tractional retinal detachment

in retinal vessels. These changes may lead to hard or soft exudates, macular oedema, and ischaemia, which leads to development of neovascularisation and the formation of fibrovascular membranes. The fibrovascular complex may contract and can result in tractional detachment of the retina. The worst complications of diabetes mellitus are TRD and VH. These complications are major causes of vision loss in patients with PDR.

Surgical management of these complications of proliferative diabetic retinopathy remains one of the most complex and challenging vitreo-retinal procedures.

The results of this study showed that PPV leads to significant vision improvement at the end of the follow-up, both in TRD and VH. Visual improvement was observed in $60 \%$ of patients and was more pronounced in the $\mathrm{VH}$ group. In a study by Quamar VA improved in 75\% of patients after PPV without tamponade [7].

The major complication associated with PPV is recurrent $\mathrm{VH}$, which causes visual impairment and reoperation. The incidence of recurrent $\mathrm{VH}$ in PDR was reported in $13-54 \%$ of cases [4-7]; in our study this incidence was $11 \%$.
In our study, re-TRD was observed in $41 \%$ and the reattachment rate was $65 \%$. In the study by Quamar the re-TRD was in 50\%, which were successfully treated by PPV without the use of an ocular tamponade in $92 \%$ [7].

Silicone oil or intraocular gases were almost routinely used after PPV for severe TRD $[8,9]$. In our study silicone oil was given as a tamponade in $66.5 \%$, air tamponade in $29 \%$, and gas tamponade in $4.5 \%$. Silicone oil enables stable retina at the time of primary vitrectomy. Moreover, using silicone oil avoids loss of vision from re-bleeds and maintains long-term tamponade in TRD.

In the present study the results of surgical treatment of VH and TRD were analysed together. In most of the studies the results are assessed in one group only.

Another important postoperative complication was elevated IOP in nine cases (20\%), Sternfeld et al. described similar findings in their retrospective studies of vitrectomised eyes [10].

The another postoperative complication is hypotony. In our study we did not document a case of hypotony. Sternfeld et al. presented $16 \%$ of patients after PPV with hypotony [10]. 
PPV is an effective treatment method of complications of PDR inter alia the TRD and VH sustained over three months. The best benefit from vitrectomy was found in type 1 diabetics, who tended to be younger and had more severe disease.

Intraoperative and postoperative complications can depend on the severity and duration of the disease, and control of the blood glucose.

In conclusion, patients with severe complications of diabetic retinopathy such as $\mathrm{VH}$ or TRD can benefit from PPV, although complications may affect the final anatomical and functional results.

\section{REFERENCES}

1. Ho T, Smiddy WE, Flynn HW. Vitrectomy in the management of diabetic eye disease. Surv Ophthalmol. 1992; 37(3): 190-202, indexed in Pubmed: 1475753.

2. Machemer $R$, Buettner $H$, Parel JM, et al. Vitrectomy: a pars plana approach. Trans Am Acad Ophthalmol Otolaryngol. 1971; 75(4): 813-820, indexed in Pubmed: 5566980.

3. O'Malley C, Heintz RM. Vitrectomy via the pars plana--a new instrument system. Trans Pac Coast Otoophthalmol Soc Annu Meet. 1972; 53: 121-137, indexed in Pubmed: 4659474.
4. Heimann K, Dahl B, Dimopoulos S, et al. Pars plana vitrectomy and silicone oil injection in proliferative diabetic retinopathy. Graefes Arch Clin Exp Ophthalmol. 1989; 227(2): 152-156, indexed in Pubmed: 2470655.

5. Newman DK. Surgical management of the late complications of proliferative diabetic retinopathy. Eye (Lond). 2010; 24(3): 441-449, doi: 10.1038/eye.2009.325, indexed in Pubmed: 20139916.

6. Oshima Y, Shima C, Wakabayashi T, et al. Microincision vitrectomy surgery and intravitreal bevacizumab as a surgical adjunct to treat diabetic traction retinal detachment. Ophthalmology. 2009; 116(5): 927-938, doi: 10.1016/j.ophtha.2008.11.005, indexed in Pubmed: 19269033.

7. Dikopf MS, Patel KH, Setlur VJ, et al. Surgical outcomes of 25-gauge pars plana vitrectomy for diabetic tractional retinal detachment. Eye (Lond). 2015; 29(9): 1213-1219, doi: 10.1038/eye.2015.126, indexed in Pubmed: 26183284.

8. Abunajma MA, Al-Dhibi $\mathrm{H}$, Abboud EB, et al. The outcomes and prognostic factors of vitrectomy in chronic diabetic traction macular detachment. Clin Ophthalmol. 2016; 10: 1653-1661, doi: 10.2147/ OPTH.S98555, indexed in Pubmed: 27616879.

9. Sternfeld A, Axer-Siegel R, Stiebel-Kalish H, et al. Advantages of diabetic tractional retinal detachment repair. Clin Ophthalmol. 2015; 9: 1989-1994, doi: 10.2147/0PTH.S90577, indexed in Pubmed: 26604667.

10. Sharma T, Fong A, Lai TY, et al. Surgical treatment for diabetic vitreoretinal diseases: a review. Clin Exp Ophthalmol. 2016; 44(4): 340-354, doi: 10.1111/ceo.12752, indexed in Pubmed: 27027299. 